\title{
Spotlight on Mogamulizumab-Kpkc for Use in Adults with Relapsed or Refractory Mycosis Fungoides or Sézary Syndrome: Efficacy, Safety, and Patient Selection
}

This article was published in the following Dove Press journal:

Drug Design, Development and Therapy

\author{
Amanda L Blackmon' \\ Lauren Pinter-Brown ${ }^{1,2}$ \\ 'Division of Hematology/Oncology, \\ Department of Medicine, University of \\ California, Irvine, Irvine, CA, USA; ${ }^{2}$ Chao \\ Family Comprehensive Cancer Center, \\ University of California, Irvine, Irvine, \\ CA, USA
}

\begin{abstract}
Advanced cutaneous T cell lymphomas (CTCL) including mycosis fungoides (MF) and Sézary syndrome (SS) are often difficult to manage once they become resistant to initial systemic treatment. Current systemic treatments usually provide a limited duration of disease control, leaving this an area in desperate need of new treatment options for better long-term control. These conditions often affect the older population where transplantation may not be a feasible option. Recent studies evaluated a novel CCR4 humanized monoclonal antibody, mogamulizumab, in relapsed/refractory MF and SS, which show a meaningful progression free survival (PFS) benefit. In August 2018, mogamulizumab was approved by the FDA for the treatment of patients with relapsed/refractory MF/SS who have failed at least one treatment. Approval was based on the Phase III MAVORIC study comparing mogamulizumab to vorinostat, an FDA approved drug for this indication, in 372 patients. In this trial, mogamulizumab was found to have a superior PFS with a median of 7.7 months compared to 3.1 months in the vorinostat arm, with a hazard ratio of $0.53, \mathrm{p}<0.001$. Mogamulizumab was well tolerated with the most common AE being infusion-related reactions (32\%), drug rash $(20 \%)$, diarrhea $(23 \%)$, and fatigue (22\%). We reviewed the literature leading to the development and approval of mogamulizumab and suggest which patients may benefit the most from this treatment.
\end{abstract}

Keywords: CTCL, mycosis fungoides, Sézary syndrome, mogamulizumab, T cell lymphoma, CCR4

\section{Introduction}

Cutaneous T cell lymphomas (CTCL) represent a heterogenous group of lymphoproliferative disorders characterized by malignant atypical clonal $\mathrm{T}$ lymphocytes found in the skin. Mycosis fungoides (MF) and Sézary syndrome (SS) are the most common subtypes of CTCL. MF is often early stage (I-IIA) at diagnosis which is by definition limited to skin involvement, often with pruritic patches and plaques on sun-protected areas such as the buttocks and breast, however there are a variety of subtypes of MF which are clinically distinct. Advanced MF is more rare (stage IIBIV), defined as tumor development, erythroderma, blood or nodal involvement, and rarely visceral involvement. Early stage MF is usually treated with skin directed therapies, however refractory early disease and advanced stage disease will often require systemic treatments for palliation, often resulting in a series of sequential
Correspondence: Lauren Pinter-Brown Email Ipinterb@uci.edu 
treatments and poor quality of life. Overall survival is dependent on stage at diagnosis, pathologic variant of $\mathrm{MF}$, age, and sex. ${ }^{1} \mathrm{SS}$ is a less common, more aggressive form of CTCL, considered a leukemic-CTCL, characterized by a distinctive erythrodermic rash with peripheral blood involvement of the malignant clonal $\mathrm{T}$ cells, with similar treatment challenges as advanced stage MF.

\section{Current Treatment for Advanced MF and Sézary Syndrome}

The systemic options for advanced disease include retinoids (bexarotene), extracorporeal photopheresis, interferon, histone deacetylase inhibitors (vorinostat and romidepsin), folate analogs (methotrexate, pralatrexate), cytotoxic chemotherapy, and now brentuximab vedotin (a CD-30 antibody-drug conjugate, however the durability of response to these agents remains relatively low and the only curative option remains allogeneic hematopoietic stem cell transplant. Patients will often receive a number of treatments in sequence, and eventually develop resistance or intolerance requiring change in treatment. The phase III MAVORIC study led to the inclusion of mogamulizumab in the armamentarium to treat the advanced stage disease.

\section{Biology of MF and SS}

MF and SS were previously thought of as a continuum of disease with SS representing the later stage form, however recent studies have shown distinct patterns of chromosomal abnormalities and phenotypic characteristics suggesting these diseases are pathologically different based on cell of origin. ${ }^{2,3}$ The clonal $\mathrm{T}$ cells on flow cytometry in SS patients are phenotypically consistent with central memory $\mathrm{T}$ cells with universal coexpression of CCR7 and L-selectin (lymph node homing molecules) as well as the differentiation marker CD27. ${ }^{2}$ In $\mathrm{MF}$ patients, rare populations of $\mathrm{T}$ cells from flow cytometry were more phenotypically consistent with skin resident effector memory $\mathrm{T}$ cells, lacking expression of CCR7/L-selectin and CD27, however displaying expression of CLA (cutaneous lymphocyte antigen, a skin homing signal receptor). Interestingly, by flow cytometry, both typically strongly express CCR4 (chemokine receptor 4; the receptor for macrophage-derived chemokine MDC and thymus and activation-regulated chemokine [TARC]), involved in cell trafficking of lymphocytes to the skin. ${ }^{2}$ Chemokines and chemokine receptors direct migration of leukocytes to certain tissues and are therefore implicated in localization of certain cell populations. The presence of CCR4 expression in $\mathrm{T}$ cell leukemia/lymphoma, has resulted in the development of a targeted anti-CCR4 humanized monoclonal antibody, mogamulizumab-kpkc (Poteligeo, Kyowa Kirin, Inc). The antibody contains a defucosylated Fc region, which results in increased antibody dependent cellular cytotoxicity (ADCC) by increased binding of CCR4 to the Fc region of the $\mathrm{IgG}$ antibody.

\section{CCR4 Expression}

Yoshie et al reported CCR4 expression in patients with Adult T cell leukemia-lymphoma (ATLL) in 2002; patients infected with HTLV-1 had outgrowth of CCR4+ T cells upon immortalization, suggesting they are either preferentially infected or confer a survival benefit for the malignant cells. ${ }^{4}$ A subgroup evaluation of 4 patients with skin involvement and ATLL, demonstrated all of these patients' skin lesions contained mRNA for CCR4, which was not found in the 3 healthy patients skin sample controls. ${ }^{4}$ CCR4 expression in ATLL has previously been associated with skin involvement and poor prognosis. ${ }^{5}$ A small study evaluating CCR4 expression by flow cytometry of peripheral blood mononuclear cells in patients with CTCL with peripheral blood involvement compared to healthy controls demonstrated $62 \%$ expression of CCR4 compared to approximately $20 \%$ in healthy volunteers. ${ }^{6}$ A subsequent study showed MF skin biopsy samples had expression of CCR4 by IHC, with $73 \%$ staining positive. ${ }^{7}$ CCR4 is also expressed on Th2 and Tregs. ${ }^{8-11}$ As a therapeutic intervention, reduction in Treg cells has been proposed for increasing antitumor immunogenicity, which would theoretically allow for more of an immune response to the abnormal/malignant T cells. ${ }^{8-11}$

\section{Approval of Mogamulizumab}

Mogamulizumab was initially approved in Japan for ATLL in 2012, and relapsed/refractory peripheral T-cell lymphoma (PTCL) and CTCL in 2014. On August 8, 2018, the FDA approved mogamulizumab for relapsed or refractory MF or SS after at least one prior therapy following the phase III MAVORIC study, as we discuss below. This may represent the first T-cell-targeted antibody therapy, similar to rituximab use in B-cell disorders. Due to the expected clinical impact of this medication, it was granted priority review, breakthrough therapy designation, and orphan drug designation. 


\section{Clinical Trial Data: Safety and Efficacy}

The Japanese approval of mogamulizumab was initially based on a Phase 2 multicenter, single arm study by Ishida et al evaluating mogamulizumab in CCR4-positive relapsed ATLL in 26 patients, with a primary end point of overall response rate (ORR) and secondary end points of best response by disease site, and overall survival. ${ }^{12}$ The ORR was $50 \%$ (including 8 complete responses [CR]), with median PFS of 5.2 months and median OS of 13.7 months. The response was different based on the site of the disease with ORR $100 \%$ (13 of 13 ; all CRs) for blood, $63 \%$ (5/8) for skin, and 25\% (3/12) for nodal and extranodal lesions. The infusion was given weekly $\times 8$ at a dose of $1 \mathrm{mg} / \mathrm{kg}$ intravenous after a previous Phase 1 study by Yamamoto et al found only $1 / 6$ patients at this dose developed DLT with grade 4 neutropenia, grade 3 skin rash and febrile neutropenia. ${ }^{13}$ This was well tolerated with the most common adverse events being infusion reactions $(89 \%)$ and skin rashes (63\%) which typically occurred after 4 or more infusions. ${ }^{12}$ Skin rash grade 2 or higher was seen in 14 patients, and of these patients, objective responses were noted in 13 patients $(93 \%)$ with 8 CR compared to patients with grade 1 or no rash reported where no response was seen. ${ }^{12}$ Skin rash was managed with topical or systemic steroids - one patient developed Stevens-Johnsons syndrome, they were, however, also receiving trimethoprim/sulfamethoxazole, fluconazole and acyclovir. ${ }^{11}$ Infusion reactions were thought to be due to the defucosylated $\mathrm{Fc}$ region on IgG1 being a more potent activator of NK cells and the release of cytokines and cytotoxic molecules. ${ }^{12}$

Follow up data has shown significant risk of refractory graft versus host disease that does not respond to steroids in patients with ATLL who underwent allogenic hematopoietic stem cell transplant following mogamulizumab treatment. Depletion of Treg cells are noted for several months following treatment, therefore mogamulizumab should be used in caution in patients who could potentially receive an allogeneic stem cell transplant. ${ }^{14,15}$

Following the above Japanese study, Phillips et al conducted a randomized Phase II trial in the US, Europe, and Latin America, evaluating the safety and efficacy of mogamulizumab versus investigators choice of chemotherapy in relapsed or refractory ATLL patients. ${ }^{16}$ The study evaluated 71 patients, $2: 1$ to receive mogamulizumab vs. investigators choice of chemotherapy. The primary endpoint was confirmed ORR, which was only $11 \%(5 / 47)$ in the mogamulizumab arm and $0 \%(0 / 24)$ in the chemotherapy arm. Ninety-six percent of these patients had CCR4 positive disease. The study evaluated disease response by compartment and found that response rate was highest in blood $(21 / 39 ; 54 \%$, all CR) and skin $(8 / 18 ; 44 \%)$. Responses by compartment to investigator's choice of chemotherapy were only seen in skin $(5 / 9,56 \%)$ and blood $(1 / 18,6 \%)$. This study had lower ORR than the previous Japanese study by Ishida et al, possibly due to different inclusion criteria - the Japanese study did not include patients with refractory disease, and the US study had more patients with poor prognostic factors, such as higher ECOG scores, older age and marrow involvement. Therefore these patients may have had a more aggressive disease at baseline. In fact, in the study by Phillips et al, $65 \%$ of patients completed $\leq 1$ treatment cycle. The criteria for response assessment may also be a factor contributing to difference in result - as a patient could have response in one compartment and progression in another with clinical benefit, yet they were considered to have progressive disease. The safety profile of mogamulizumab was consistent with the prior study with the most common adverse events being infusion reactions and skin eruptions.

In 2014, the approval for mogamulizumab in Japan was expanded to relapsed/refractory peripheral T-cell lymphoma and CTCL following a single arm, open label, multicenter phase II study by Ogura et al evaluating the use of mogamulizumab in CCR-4 positive relapsed peripheral T cell lymphoma (PTCL) and CTCL. ${ }^{17}$ Patients refractory to the last line of treatment were excluded. The primary end point was the ORR, and the secondary end points included safety, progression-free survival (PFS), and overall survival (OS). 64 patients were screened with histologically confirmed PTCL or CTCL, and of these patients, 50/64 (78\%) were CCR-4 positive (and therefore eligible). Thirty-eight of these patients met further eligibility criteria (ECOG $\leq 2$, age $\geq 20$, adequate neutrophils, hemoglobin and platelets, adequate hepatic and kidney function, without CNS involvement or bulky adenopathy requiring radiotherapy, without prior allogeneic stem cell transplant, active infection including hepatitis $\mathrm{B}$ or $\mathrm{C}$, or HIV). Of the 38 eligible patients, the response rate was $35 \%$ (13/37, CI, 20-53\%), with 5 patients (14\%) obtaining a CR. The median PFS was 3 months (CI 1.6-4.9 months). In PTCL, the ORR was $34 \%$ (10/29, CI $18-54 \%)$, and in CTCL ORR was $38 \%(3 / 8$, CI $9-76 \%)$ in CTCL. In patients that did have a response (13/37), the median 
PFS was 5.5 months. Adverse events were consistent with prior studies - including lymphopenia, infusion reactions, and treatment related skin disorders.

Following the Japanese expansion of approval, Duvic et al performed an open-label, multicenter, phase $1 / 2$ study in the US to evaluate the safety and efficacy of mogamulizumab in previously treated patients with CTCL (limited to $\mathrm{MF}$ or SS). ${ }^{18}$ The MTD was not reached in the Phase I dose escalation portion of this study, therefore in agreement with the Japanese phase I data, a dose of $1 \mathrm{mg} / \mathrm{kg}$ became the recommended dose for phase 2 . Mogamulizumab was dosed at $1 \mathrm{mg} / \mathrm{kg}$ IV weekly $\times 4$ administered intravenously over 1 hour, then every 2 weeks until disease progression. The primary end point of the single arm phase 2 component was ORR; the secondary end points were response duration, and time to progression. Patients with disease involvement of the central nervous system, autoimmune conditions, HIV/hepatitis, uncontrolled infection, class III or IV heart disease, and uncontrolled diabetes were excluded. CCR4 expression was not required for eligibility, however was evaluated by multicolor flow cytometry, which showed $63.8 \%$ of patients with positive disease, including all patients with blood involvement. Thirty-eight patients were evaluated, with ORR of $36.8 \%$ (14/38, CI 21.8-54\%); the ORR was higher in SS patients, $47.1 \%(n=17)$ than MF, $28.6 \%$ $(n=21)$. Median time to response (TTR) was 31.5 days (range, 26-154). Median progression-free survival was 11.4 months and median duration of response was 10.4 months. Of 19 patients with disease in the blood $(\geq \mathrm{B} 1)$ who were positive for CCR4 expression by flow cytometry, 18 had a response in blood (95\%) and there were 11 CRs. Response in the blood was seen in the first 4 weeks and persisted for up to 3 years.

The median age of patients was 66 years, with median of 3 prior lines of treatment. ${ }^{18}$ Assessment methods for response were based on location of disease: modified Severity Weighted Assessment Tool was used for quantitative assessment of disease burden in the skin of all patients, flow cytometry was used to monitor blood involvement, and PET scans were used to evaluate lymphatic or visceral disease. An overall global response score included assessment of all compartments - skin, lymph nodes, blood and viscera (the Olsen criteria). ${ }^{19}$ The most common adverse events were nausea (31\%), chills $(23.8 \%)$, headache $(21.4 \%)$, and infusion reactions $(21.4 \%)$; most events were grade $1 / 2$ and there were no significant hematologic effects. A total of 7 patients (16.7\%) experienced a drug eruption rash, and all were withdrawn from the study. This study led to the phase III MAVORIC trial.

The FDA approval of mogamulizumab for CTCL (MF and SS) that has failed at least one treatment line followed the phase III MAVORIC trial by Kim et al, evaluating mogamulizumab versus vorinostat in previously treated CTCL. ${ }^{20}$ The study was an open label, multi-center, international, randomized controlled trial in adults $>18$-yearsold with relapsed/refractory CTCL who failed at least one line of treatment. The primary end point was progression free survival (PFS) by investigator assessment. Eligible patients were stage IB-IVB, with histologically confirmed MF or SS, with ECOG of 1 or less, and adequate hematologic, renal and hepatic function. Patients were assigned 1:1 to receive mogamulizumab at $1 \mathrm{mg} / \mathrm{kg}$ weekly by intravenous infusion $\times 4$ over 1 hour, for the first 28-day cycle, followed by days 1 and 15 of subsequent cycles, or vorinostat $400 \mathrm{mg}$ daily. The study allowed for a single arm cross over from vorinostat to mogamulizumab if there was progression observed in 2 consecutive cycles or intolerable toxicity despite dose reduction or appropriate management. No dose reductions were allowed in the mogamulizumab arm. Patients continued treatment until progression or toxicity - or if the patient had a global CR, for a total of 12 months, whichever came first. Similar to the previous phase I/II trial, patients with disease involvement of the central nervous system, autoimmune disease, and active illness were excluded. Patients on stable low dose steroids were permitted to stay on the study. In MAVORIC, patients were also excluded who had large cell transformation, prior allogenic transplant, as were patients with a CD4 count less than 200 after treatment with alemtuzumab. Importantly, CCR4-positivity was not required for study eligibility, however, was looked at in post hoc analysis. Response to treatment/stable disease was evaluated in a similar fashion to the phase I/II study with 4 disease compartments - skin with mSWAT, nodal and visceral disease by $\mathrm{CT}$, and blood involvement by flow cytometry. ${ }^{20}$

Three hundred seventy patients were evaluated (204 with MF and 168 with SS), two patients withdrew consent prior to receiving medication that were randomized to the mogamulizumab arm. The median age in MAVORIC trial was 65 and median number of prior treatments was 3 . Mogamulizumab had superior PFS with a median of 7.7 months (95\% CI 5.7-10.3) compared to 3.1 months (95\% CI 2.9-4.1) in the vorinostat group - hazard ratio of 0.53 , $\mathrm{p}<0.001$. The investigator assessed that the ORR was higher in the mogamulizumab group at $28 \%(52 / 186, \mathrm{CI}$ 
$21.6-35 \%)$ compared to vorinostat at $5 \%(9 / 186$, CI $2.2-9 \%$ ). Interestingly, the ORR for vorinostat was lower than prior single arm phase IIB study by Olsen et al evaluating the efficacy of vorinostat in relapsed/refractory CTCL (29.7\%, CI 19.7-41.5\%), though in that study, evaluation was by skin response only, which was more comparable with MAVORIC data in the skin compartment $\left(16 \%\right.$ overall response).$^{20,21 \text {, }}$

The response by disease (MF or SS) and compartment involved also varied; patients with MF had a $21 \%$ ORR in the mogamulizumab arm compared to $7 \%$ in the vorinostat arm; patients with SS had 37\% ORR in the mogamulizumab arm compared to $2 \%$ in the vorinostat arm. The duration of response also varied by disease. Patients with MF had a median duration of response of 13.1 months (4.7-18) in the mogamulizumab arm, compared to 9.1 months (5.6 - not estimable) in the vorinostat arm. Patients with SS had a median duration of response of 17.3 months (9.4-19.9) in the mogamulizumab arm compared to 6.9 months (6.9-6.9) in the vorinostat arm. Response by compartment was also notably different between the treatment arms. In the blood compartment, patients in the mogamulizumab arm had a $68 \%$ response rate compared to $19 \%$ in the vorinostat arm. In the skin compartment, patients in the mogamulizumab arm had a $42 \%$ response rate compared to $16 \%$ in the vorinostat arm. In the lymph node compartment, patients in the mogamulizumab arm had a $17 \%$ response rate compared to $4 \%$ in the vorinostat arm. Of the small number of patients with visceral disease - no patients in either treatment arm had a response in this compartment. Of note, the response in nodal involvement is relatively low in both arms.

The post hoc analysis of median TTR was 3.3 months in the mogamulizumab group and 5.1 months in the vorinostat group. Interestingly, TTR also varied by compartment. In the mogamulizumab arm - median TTR in blood was 1.1 months (CI 1-1.2), in skin 3.0 months (CI 1.9-4.7), in lymph nodes 3.3 months (CI 2.8-6.8).

Out of 186 patients assigned to the vorinostat arm, 136 crossed over to receive mogamulizumab (109 after progression and 27 after drug toxicity). The median time to cross over was 3.16 months (0.7-24.3) - this may represent early termination of treatment with vorinostat given the median TTR was 5.1 months, however in the pivotal vorinostat trial, the median TTR was 56 days. ${ }^{22}$ Hence, a cross over time over 3 months should have allowed any responses to be noted, if they were going to occur. The response rate of the patients who crossed over was $31 \%$ (41/133) with post hoc analysis showing median PFS from starting mogamulizumab was 8.9 months (5.4-14.8). This represents a similar PFS in patients pretreated with vorinostat to patients that were initially randomized to the investigational arm.

An exploratory post hoc analysis in the MAVORIC trial looked at 290 evaluable skin samples, of which 280 had positive CCR 4 expression status defined by $10 \%$ infiltrating lymphoid cells. Despite most patients expressing CCR4, there was no apparent difference in the patients who did or did not have CCR4-positivity and overall response. In preclinical data, in vitro CCR4 was downregulated in cell lines exposed to HDAC inhibitors, suggesting the possibility of reduced efficacy of mogamulizumab subsequently, however the MAVORIC trial suggests otherwise. ${ }^{23}$

The most common grade 1-2 adverse events in the mogamulizumab group were infusion related reactions $(32 \%)$, drug rash $(20 \%)$, diarrhea $(23 \%)$, and fatigue (22\%); compared to the vorinostat group with diarrhea $(57 \%)$, nausea (41\%), fatigue (32\%) and thrombocytopenia (34\%). Similar reports of grade 3-4 adverse events were present in each group - 75/184 in the mogamulizumab group and 76/186 in the vorinostat group. The most common grade 3-4 adverse events in the mogamulizumab group were pyrexia in 8 patients $(4 \%)$ and cellulitis in 5 patients $(3 \%)$. In the vorinostat group, cellulitis was reported in 6 patients $(3 \%)$, pulmonary embolism in 6 patients $(3 \%)$, and sepsis in 5 patients $(3 \%)$.

Treatment-related serious adverse events (SAE) were reported in 36/184 patients in mogamulizumab group compared to 30/186 in the vorinostat group. The most common treatment related SAE in the mogamulizumab group were pneumonia in 4 patients (2\%) and pyrexia in 4 patients $(2 \%)$. In the vorinostat group, treatment related SAE were pulmonary embolism in 5 patients (3\%) and thrombocytopenia in 3 patients (2\%). There were 12 deaths out of 372 total patients $(3 \%)-3 / 184(2 \%)$ in the mogamulizumab group, two of which were related to treatment - sepsis and polymyositis, and one with unrelated disease progression. In the vorinostat group there were 9 deaths out of 186 patients $(5 \%)$, three of which were related to treatment, two pulmonary emboli and one bronchopneumonia - the other 6 unrelated deaths were due to disease progression, intestinal obstruction, sepsis, endocarditis, pneumonia, and depressed level of consciousness with skin disorder. 


\section{Consideration for Using Mogamulizumab in Standard Practice}

Mogamulizumab is a new feasible option in the older population based on the MAVORIC trial above with a median age of 65 and with a median of 3 prior lines of treatment - if they are ECOG 1 or less, with adequate hematologic, renal and hepatic function and without the exclusion criteria mentioned above. One important consideration will be in the younger, fitter population with an aggressive disease where allogeneic transplant may be considered. Prior treatment with mogamulizumab may increase the risk of refractory GVHD following transplantation, therefore the timing of mogamulizumab relative to the transplant and/or GVHD prophylaxis should be carefully considered.

Mogamulizumab has shown the most impressive results in patients with blood and/or skin involvement with advanced MF or SS. The results of the studies mentioned above are also summarized in Table 1 for comparison. It is not surprising that patients with SS, with skin and blood involvement by definition, also have a better response to mogamulizumab. The response in nodal and visceral disease was not as robust, suggesting these patients may be treated with other appropriate options as mentioned above. Based on the efficacy of mogamulizumab in clearing disease involvement of the blood and skin compartments, we would use mogamulizumab prior to vorinostat in patients with blood involvement. Patients with large cell transformation were also excluded from the Phase 3 study likely due to the aggressive nature of the disease, possibly indicating the need for more aggressive treatment. Only two patients developed LCT while on MAVORIC therefore further observations may be necessary to determine if LCT is seen more commonly in mogamulizumab treated patients.

Lastly, the use of mogamulizumab in patients with autoimmune disease is relatively contraindicated because of the mechanism of action and serious grade 3 or higher adverse events previously reported in patients largely with ATLL including possible immune mediated myositis, myocarditis, polymyositis, hepatitis, pneumonitis, Guillain-barre, polymyalgia rheumatic and hypothyroidism. ${ }^{24}$ As previously mentioned, there is a theoretical risk of depleting Treg cells that express CCR4, responsible for mitigating an overactive immune response, and therefore unleashing the immune system on malignant cells/lesions or perhaps off target in autoimmune conditions.

Table I Response to Mogamulizumab by Disease Compartment

\begin{tabular}{|c|c|c|c|c|c|}
\hline Disease & Study & $\begin{array}{l}\text { ORR to } \\
\text { Moga vs. } \\
\text { Comparator }\end{array}$ & $\begin{array}{l}\text { ORR in Blood } \\
\text { Compartment to } \\
\text { Moga vs. Comparator }\end{array}$ & $\begin{array}{l}\text { ORR in Skin } \\
\text { Compartment to } \\
\text { Moga vs. } \\
\text { Comparator }\end{array}$ & $\begin{array}{l}\text { ORR in Nodal/Extranodal } \\
\text { Compartment to Moga vs. } \\
\text { Comparator }\end{array}$ \\
\hline $\begin{array}{l}\text { Relapsed } \\
\text { ATLL }\end{array}$ & $\begin{array}{l}\text { Ishida et al; Phase II } \\
\text { single arm } \\
\text { Mogamulizumab }\end{array}$ & $\begin{array}{l}50 \%(13 / 26) \\
\text { Including } 8 \\
\mathrm{CR}\end{array}$ & $100 \%(13 / 13)$ & $63 \%(5 / 8)$ & $35 \%(3 / / 2)$ \\
\hline $\begin{array}{l}\text { Relapsed/ } \\
\text { Refractory } \\
\text { ATLL }\end{array}$ & $\begin{array}{l}\text { Phillips et al; Phase } \\
\text { II Mogamulizumab } \\
\text { vs. chemo }\end{array}$ & $\begin{array}{l}\text { I I \% (5/47) vs. } \\
0 \%(0 / 24)\end{array}$ & $\begin{array}{l}54 \%(2 \mathrm{I} / 39) ; \text { all } \mathrm{CR} \text { vs. } \\
6 \%(1 / 18)\end{array}$ & $\begin{array}{l}44 \%(8 / 18) \\
\text { vs. } \\
56 \%(5 / 9)\end{array}$ & $\begin{array}{l}7 \%(3 / 44) \\
\text { vs. } \\
0 \%(0 / 22)\end{array}$ \\
\hline $\begin{array}{l}\text { Relapsed/ } \\
\text { Refractory } \\
\text { PTCL and } \\
\text { CTCL }\end{array}$ & $\begin{array}{l}\text { Ogura et al; Phase II } \\
\text { single arm } \\
\text { Mogamulizumab }\end{array}$ & $\begin{array}{l}35 \%(13 / 37) \\
\text { Including } 5 \\
\text { CR }\end{array}$ & Not reported & $58 \%(7 / / 2)$ & $33 \%(11 / 33)$ \\
\hline $\begin{array}{l}\text { Relapsed/ } \\
\text { Refractory } \\
\text { CTCL }\end{array}$ & $\begin{array}{l}\text { Duvic et al; Phase I/ } \\
\text { II single arm } \\
\text { Mogamulizumab }\end{array}$ & $37 \%(\mid 5 / 4 I)$ & $95 \%$ (I8/I9); II were CR & $42 \%(16 / 38)$ & $25 \%(7 / 28)$ \\
\hline $\begin{array}{l}\text { Relapsed/ } \\
\text { Refractory } \\
\text { CTCL }\end{array}$ & $\begin{array}{l}\text { MAVORIC } \\
\text { Kim et al; Phase III } \\
\text { Mogamulizumab vs. } \\
\text { Vorinostat }\end{array}$ & $\begin{array}{l}28 \%(52 / 186) \\
\text { vs } 5 \%(9 / 186)\end{array}$ & $\begin{array}{l}68 \%(83 / / 22) \text { vs. } \\
19 \%(23 / / 23)\end{array}$ & $\begin{array}{l}42 \%(78 / 86) \text { vs. } \\
16 \%(29 / 186)\end{array}$ & $\begin{array}{l}17 \%(2|/| 24) \\
\text { vs. } \\
4 \%(5 / \mid 22)\end{array}$ \\
\hline
\end{tabular}

Notes: Overall response rate and disease compartment response by study: Highest response rates consistently seen in the blood and skin compartments. 


\section{Conclusion}

The MAVORIC trial used PFS as the primary end point in order to capture a duration of disease control which may be more meaningful than response rate previously used in other studies, in this patient population with chronic, incurable disease. The MAVORIC trial and prior studies on mogamulizumab support this drug as a safe and effective treatment for MF/SS where treatments with durable responses are clearly needed. The safety profile seen in the phase III study was similar to the prior phase I/II without new safety concerns. The approval of mogamulizumab adds an efficacious and well tolerated drug to the current armoire of sequential drugs available for patients with advanced MF and SS, especially with skin and/or blood involvement.

\section{Disclosure}

Dr Lauren Pinter-Brown was part of the advisory board for Kiowa Kirin during the conduct of the study. The authors report no other conflicts of interest in this work.

\section{References}

1. Kim YH, Liu HL, Mraz-Gernhard S, Varghese A, Hoppe RT. Longterm outcome of 525 patients with mycosis fungoides and sézary syndrome: clinical prognostic factors and risk for disease progression. Arch Dermatol. 2003;139(7):857-866. doi:10.1001/archderm. 139.7.857

2. Campbell JJ, Clark RA, Watanabe R, Kupper TS. Sezary syndrome and mycosis fungoides arise from distinct T-cell subsets: a biologic rationale for their distinct clinical behaviors. Blood. 2010;116 (5):767-771. doi:10.1182/blood-2009-11-251926

3. van Doorn R, van Kester MS, Dijkman R, et al. Oncogenomic analysis of mycosis fungoides reveals major differences with Sezary syndrome. Blood. 2009;113(1):127-136. doi:10.1182/blood-2008-04-153031

4. Yoshie O, Fujisawa R, Nakayama T, et al. Frequent expression of CCR4 in adult T-cell leukemia and human T-cell leukemia virus type 1-transformed T cells. Blood. 2002;99:1505-1511. doi:10.1182/blood. V99.5.1505

5. Ishida T, Utsunomiya A, Iida S, et al. Clinical significance of CCR4 expression in adult T-cell leukemia/lymphoma: its close association with skin involvement and unfavorable outcome. Clin Cancer Res. 2003;9(10 Pt 1):3625-3634.

6. Ferenczi K, Fuhlbrigge RC, Pinkus J, Pinkus GS, Kupper TS. Increased CCR4 expression in cutaneous $\mathrm{T}$ cell lymphoma. $J$ Invest Dermatol. 2002;119(6):1405-1410. doi:10.1046/j.1523-1747.2002.19610.x

7. Duvic M, Pinter-Brown L, Foss F, et al. Correlation of target molecule expression and overall response in refractory cutaneous T-cell lymphoma patients dosed with mogamulizumab (KW-0761), a monoclonal antibody directed against CC chemokine receptor type 4 (CCR4). Blood. 2012;120 (21):3697. doi:10.1182/blood.V120.21.3697.3697

8. Ishida T, Ueda: R. CCR4 as a novel molecular target for immunotherapy of cancer. Cancer Sci. 2006;97:1139-1146. doi:10.1111/j.13497006.2006.00307.x

9. Xiao N, Jorgensen JL, Goswami M, et al. Reduction of regulatory $\mathrm{T}$ cells by mogamulizumab, a defucosylated anti-cc chemokine receptor 4 antibody, in patients with aggressive/refractory mycosis fungoides and sézary syndrome. Clin Cancer Res. 2015;21(2).
10. Iellem A, Mariani M, Lang R, et al. Unique chemotactic response profile and specific expression of chemokine receptors CCR4 and CCR8 by $\mathrm{CD} 4+\mathrm{CD} 25+$ regulatory $\mathrm{T}$ cells. $J$ Exp Med. 2001;194:847-853. doi:10.1084/jem.194.6.847

11. Andrew DP, Ruffing N, Kim CH, et al. C-C chemokine receptor 4 expression defines a major subset of circulating nonintestinal memory T cells of both Th1 and Th2 potential. J Immunol. 2001;166:103-111. doi:10.4049/jimmunol.166.1.103

12. Ishida $\mathrm{T}$, Joh $\mathrm{T}$, Uike $\mathrm{N}$, et al. Defucosylated anti-CCR4 monoclonal antibody (KW-0761) for relapsed adult T-cell leukemia-lymphoma: a multicenter phase II study. J Clin Oncol. 2012;30:837-842. doi:10.1200/JCO.2011.37.3472

13. Yamamoto K, Utsunomiya A, Tobinai K, et al. Phase I study of KW-0761, a defucosylated humanized anti-CCR4 antibody, in relapsed patients with adult T-cell leukemia-lymphoma and peripheral T-cell lymphoma. J Clin Oncol. 2010;28(9):1591-1598. doi:10.1200/JCO.2009.25.3575

14. Fuji S, Inoue Y, Utsunomiya A, et al. Pretransplantation anti-CCR4 antibody mogamulizumab against adult t-cell leukemia/lymphoma is associated with significantly increased risks of severe and corticosteroid-refractory graft-versus-host disease, nonrelapse mortality, and overall mortality. J Clin Oncol. 2016;34(28):3426-3433. doi:10.1200/JCO.2016.67.8250

15. Sugio T, Kato K, Aoki T, et al. Mogamulizumab treatment prior to allogeneic hematopoietic stem cell transplantation induces severe acute graft-versus-host disease. Biol Blood Marrow Transplant. 2016;22(9):1608-1614. doi:10.1016/j.bbmt.2016.05.017

16. Phillips AA, Fields PA, Hermine O, et al. Mogamulizumab versus investigator's choice of chemotherapy regimen in relapsed/refractory adult T-cell leukemia/lymphoma. Haematologica. 2019;104(5):9931003. doi:10.3324/haematol.2018.205096

17. Ogura M, Ishida T, Hatake K, et al. Multicenter phase II study of mogamulizumab (KW-0761), a defucosylated anti-cc chemokine receptor 4 antibody, in patients with relapsed peripheral T-cell lymphoma and cutaneous T-cell lymphoma. J Clin Oncol. 2014;32 (11):1157-1163. doi:10.1200/JCO.2013.52.0924

18. Duvic M, Pinter-Brown LC, Foss FM, et al. Phase $1 / 2$ study of mogamulizumab, a defucosylated anti-CCR4 antibody, in previously treated patients with cutaneous T-cell lymphoma. Blood. 2015;125:1883-1889. doi:10.1182/blood-2014-09-600924

19. Olsen EA, Whittaker S, Kim YH. Clinical end points and response criteria in mycosis fungoides and Sézary syndrome: a consensus statement of the International Society for Cutaneous Lymphomas, the United States Cutaneous Lymphoma Consortium, and the Cutaneous Lymphoma task force of the European Organisation for Research and Treatment of Cancer. J Clin Oncol. 2011;29 (18):2598-2607. doi:10.1200/JCO.2010.32.0630

20. Kim YH, Bagot M, Pinter-Brown L, et al. Mogamulizumab versus vorinostat in previously treated cutaneous T-cell lymphoma (MAVORIC): an international, open-label, randomised, controlled phase 3 trial. Lancet Oncol. 2018;19(9):1192-1204. doi:10.1016/ S1470-2045(18)30379-6

21. Olsen EA, Whittaker S, Kim YH, et al. Phase IIb multicenter trial of vorinostat in patients with persistent, progressive or treatment refractory cutaneous T-cell lymphoma. Blood. 2015;25:1883-1889.

22. Olsen EA, Kim YH, Kuzel TM, et al. Phase IIb multicenter trial of vorinostat in patients with persistent, progressive, or treatment refractory cutaneous T-cell lymphoma. J Clin Oncol. 2007;25 (21):3109-3115. doi:10.1200/JCO.2006.10.2434

23. Kitadate A, Ikeda S, Abe F, et al. Histone deacetylase inhibitors downregulate CCR4 expression and decrease mogamulizumab efficacy in CCR4-positive mature T-cell lymphomas. Haematologica Jan. 2018;103(1):126-135. doi:10.3324/haematol. 2017.177279

24. Mogamulizumab [Package Insert]. Bedminster, NJ: Kyowa Kirin, Inc; 2018. 


\section{Publish your work in this journal}

Drug Design, Development and Therapy is an international, peerreviewed open-access journal that spans the spectrum of drug design and development through to clinical applications. Clinical outcomes, patient safety, and programs for the development and effective, safe, and sustained use of medicines are a feature of the journal, which has also

been accepted for indexing on PubMed Central. The manuscript management system is completely online and includes a very quick and fair peer-review system, which is all easy to use. Visit http://www. dovepress.com/testimonials.php to read real quotes from published authors.

Submit your manuscript here: https://www.dovepress.com/drug-design-development-and-therapy-journal 